\title{
Comparative Effectiveness and Safety of Low-Dose Oral Anticoagulants in Patients With Atrial Fibrillation
}

OPEN ACCESS

Edited by:

Mina Tadrous,

Women's College Hospital, Canada

Reviewed by:

Johannes P. Mouton,

University of Cape Town, South Africa

Eva Kline-Rogers,

University of Michigan, United States

*Correspondence:

Sylvie Perreault

sylvie.perreault@umontreal.ca

Specialty section:

This article was submitted to

Experimental Pharmacology and Drug

Discovery,

a section of the journal

Frontiers in Pharmacology

Received: 09 November 2021

Accepted: 28 December 2021

Published: 14 January 2022

Citation:

Perreault $S$, Dragomir A, Côté $R$, Lenglet $A$, de Denus $S$, Dorais $M$,

White-Guay B, Brophy J,

Schnitzer ME, Dubé M-P and

Tardif J-C (2022) Comparative

Effectiveness and Safety of Low-Dose Oral Anticoagulants in Patients With

Atrial Fibrillation.

Front. Pharmacol. 12:812018.

doi: 10.3389/fphar.2021.812018

\begin{abstract}
Sylvie Perreault ${ }^{1 \star}$, Alice Dragomir ${ }^{2}$, Robert Côté $^{3}$, Aurélie Lenglet ${ }^{4,5}$, Simon de Denus ${ }^{1,6}$, Marc Dorais ${ }^{7}$, Brian White-Guay ${ }^{8}$, James Brophy ${ }^{9}$, Mireille E. Schnitzer ${ }^{1}$, Marie-Pierre Dubé ${ }^{6,8}$ and Jean-Claude Tardif ${ }^{6,8}$

${ }^{1}$ Faculty of Pharmacy, Université de Montréal, Montreal, QC, Canada, ${ }^{2}$ Department of Urology, Faculty of Medicine, University McGill, Montreal, QC, Canada, ${ }^{3}$ Faculty of Medicine, Department of Neurology and Neurosurgery, McGill University, Montreal, QC, Canada, ${ }^{4}$ Laboratory MP3CV, Faculty of Pharmacy, University of Picardie Jules Verne, Amiens, France, ${ }^{5}$ Pharmacy, Amiens University Medical Center, Amiens, France, ${ }^{6}$ Montreal Heart Institute, Montreal, QC, Canada, ${ }^{7}$ StatSciences Inc., Notre-Damede-l'Île-Perrot, QC, Canada, ${ }^{8}$ Faculty of Medicine, Université de Montréal, Montreal, QC, Canada, ${ }^{9}$ Faculty of Medicine, McGill University, Montreal, QC, Canada
\end{abstract}

Aims: Observational studies of various dose levels of direct oral anticoagulants (DOACs) for patients with atrial fibrillation (AF) found that a high proportion of patients received a dose lower than the target dose tested in randomized controlled trials. There is a need to compare low-dose DOACs with warfarin or other DOACs on effectiveness and safety.

Methods: Using administrative data from Quebec province, Canada, we built a cohort of new warfarin or DOAC users discharged from hospital between 2011 and 2017. We determined $\mathrm{CHA}_{2} \mathrm{DS}_{2}$-VASc and HAS-BLED scores, and comorbidities for 3-year prior cohort entry. The primary effectiveness endpoint was a composite of ischemic stroke/ systemic embolism (SE), and secondary outcomes included a safety composite of major bleeding (MB) events and effectiveness composite (stroke/SE, death) at 1-year follow-up. We contrasted each low-dose DOAC with warfarin or other DOACs as references using inverse probability of treatment weighting to estimate marginal Cox hazard ratios (HRs).

Results: The cohort comprised 22,969 patients (mean age: 80-86). We did not find a significant risk reduction for the stroke/SE primary effectiveness endpoint for DOACs vs. warfarin; however, we observed a significantly lower risk for low-dose dabigatran vs. warfarin (HR [95\% Cl]: 0.59 [0.42-0.81]) for effectiveness composite, mainly due to a lower death rate. The differences in effectiveness and safety composites between low-dose rivaroxaban vs. warfarin were not significant. However, low-dose apixaban had a better safety composite (HR: 0.68 [0.53-0.88]) vs. warfarin. Comparisons of dabigatran vs. apixaban showed a lower risk of stroke/SE (HR: 0.53 [0.30-0.93]) and a 2-fold higher risk of MB. The MB risk was higher for rivaroxaban than for apixaban (HR: 1.58 [1.09-2.29]).

Conclusions: The results of this population-based study suggest that low-dose dabigatran has a better effective composite than warfarin. Compared with apixaban, low-dose dabigatran had a better effectiveness composite but a worse safety profile. Lowdose apixaban had a better safety composite than warfarin and other low-dose DOACs. 


\section{Given that the comparative effectiveness and safety seem to vary from one DOAC to} another, pharmacokinetic data for specific populations are now warranted.

Keywords: atrial fibrillation, oral anticoagulant, effectiveness outcomes, safety outcomes, low dose

\section{INTRODUCTION}

Atrial fibrillation (AF) is known to cause embolic stroke, and the prevalence of AF is likely to increase (Colilla et al., 2013). Ischemic strokes associated with $\mathrm{AF}$ are more severe and more lethal than strokes in the absence of AF (McGrath et al., 2013). Oral anticoagulant (OAC) therapy with direct oral anticoagulants (DOACs, such as dabigatran, rivaroxaban, apixaban, and edoxaban) or vitamin $\mathrm{K}$ antagonists (e.g., warfarin) can effectively prevent ischemic events (including strokes) in patients with non-valvular AF (Hart et al., 2007; Culebras and Messé, 2014; January et al., 2014; Lip et al., 2018). The optimal use of warfarin becomes more difficult in older adults, since the latter have a greater risk of both thromboembolic and bleeding events (Samsa and Matchar, 2000; Fanikos et al., 2005; Miyasaka et al., 2006). The difficulties associated with warfarin use have led to the widespread acceptance of fast-acting DOACs, which target specific clotting factors. DOACs are associated with a lower risk of drug interactions, are less influenced by dietary factors, and constitute alternatives to warfarin for the prevention of stroke and systemic embolism (SE) in patients with non-valvular AF (January et al., 2014; Lip et al., 2018).

The use of DOACs in patients with non-valvular AF has been studied in large randomized controlled trials (RCTs) (Connolly et al., 2009; Granger et al., 2011; Patel et al., 2011; Xu et al., 2016). Compared with warfarin, DOACs were shown to be superior or comparable in terms of efficacy and had similar or lower bleeding rates-especially for intracranial hemorrhage (Connolly et al., 2009; Granger et al., 2011; Patel et al., 2011; Xu et al., 2016). Recent real-world, populationbased studies of DOAC use by patients with AF (Maura et al., 2017; Perreault et al., 2020) found that a low dose was more prevalent than the standard dose used in RCTs (Perreault et al., 2020).

Extrapolating the RCT data on DOAC doses to clinical decision-making is limited by the small number of patients included in RCTs (Connolly et al., 2009; Connolly et al., 2010; Granger et al., 2011; Patel et al., 2011). Variability in treatment adherence and patient follow-up constitutes an additional challenge in clinical management and is not optimally reflected by the RCT results (Steinberg et al., 2013; Cutler et al., 2014). Hence, there is a need to compare various lowdose DOACs with warfarin and each other in terms of effectiveness and safety in patients with AF. To address this gap in our knowledge, we built a cohort of hospitalized patients with a primary or secondary diagnosis of AF and then compared low-dose DOACs with warfarin and with each other.

\section{MATERIALS AND METHODS}

\section{Data Source}

We built a cohort using data in the Med-Echo administrative databases (hospital discharges), medical services, and public drug plans administered by the Régie de l'Assurance Maladie du Québec (RAMQ). The databases were linked using encrypted health insurance numbers. Information from these databases provides a complete picture of hospital admissions (Tamblyn et al., 1995; Tamblyn et al., 2000; Wilchesky et al., 2004; Eguale et al., 2010). The protocol was approved by an independent ethics committee at the University of Montreal.

\section{Population-Based Cohort}

The cohort was designed using claims data from the Quebec RAMQ and Med-Echo databases. We identified adult patients with a primary or secondary diagnosis of AF (inpatient codes: ICD-9 427.3, 427.31, or 427.32 or ICD-10 I48) discharged alive from hospital into the community between January 1st, 2011, and December 31st, 2017 (Humphries et al., 2004; Perreault et al., 2018). For patients with multiple admissions with an AF diagnosis, only the first admission was analyzed. In previous validation studies, the diagnostic performance of ICD-9 codes for $\mathrm{AF}$ was relatively good, with median positive predictive values of over $80 \%$ (Jensen et al., 2012).

We next identified patients who had filled a new prescription of apixaban ( $2.5 \mathrm{mg}$ twice daily), dabigatran (110 $\mathrm{mg}$ twice daily), rivaroxaban (15 mg once daily) or warfarin in the 12 months following hospital discharge. These new users had not been exposed to any OACs in the year before the index claim date. Eligible patients also had to have continuous health insurance coverage for at least 12 months before the index claim date. The date of the first OAC claim after hospital discharge was taken as the date of cohort entry.

We excluded patients with end-stage chronic kidney disease or a kidney transplant, patients on dialysis at any time in the 3 years before the index date, those having undergone hip or knee replacement surgery in the 6 weeks before the index date, and those with a diagnosis of deep vein thrombosis or pulmonary embolism at baseline. We also excluded patients with a coagulation deficiency or having undergone certain medical procedures (including cardiac catheterization, stent placement, a coronary artery bypass graft, medical procedures for cerebrovascular disease, or defibrillator implantation) in the 3 months prior to the index date. Lastly, we excluded patients having undergone a cardiac valvular replacement in the 5 years prior to cohort entry.

\section{Exposure to Oral Anticoagulants}

We used fill dates and the number of days' supply per prescription to establish the dates of the patients' exposure to DOACs or warfarin. Patients were categorized as being on treatment if they had filled prescriptions within 30 days of the end of the previous treatment period. A gap of 30 days or less between treatments was allowed; this is a reasonable duration because of the DOACs' short half-life in vivo (Perreault et al., 2020). Consequently, we 
chose 1 month as the allowable gap, which corresponds to an adherence of $92 \%$ or more over the fixed 12-month exposure assessment period.

\section{Outcomes}

The primary effectiveness outcome was a primary diagnosis of ischemic stroke or systemic embolism (SE) after hospital admission for acute care during the 12-month follow-up period. The secondary outcomes were 1) a safety composite of major bleeding events (intracranial hemorrhage (ICH), gastrointestinal hemorrhage, and all other bleeding events), 2) a benefit/risk composite (stroke/SE, major bleeding, and all-cause mortality), 3) all-cause mortality, 4) an effectiveness composite (stroke/SE and all-cause mortality), and 5) major bleeding (intracranial hemorrhage and gastrointestinal bleeding only) over the same period of follow-up.

We identified outcomes using ICD-9 or ICD-10 codes for the primary diagnosis of inpatient claims (Supplementary Table S1). The positive predictive values were over $80 \%$ (Levy et al., 1999; Blais et al., 2012). These codes performed relatively well in previous validation studies (Tirschwell and Longstreth, 2002; Blais et al., 2012; Jensen et al., 2012; Thigpen et al., 2015). The definition of major bleeding has been published previously (Perreault et al., 2018).

\section{Demographic and Clinical Characteristics of the Study Population}

We documented the demographic data at cohort entry. Social and economic deprivation was assessed using the Pampalon index (Pampalon et al., 2009). We determined the presence of comorbidities from specific ICD-9 or ICD-10 codes recorded during the hospital stay and those recorded for inpatient and outpatient diagnoses during the 3 years prior to the index date (Blais et al., 2012; Roy et al., 2020). Using the data on patient characteristics and associated comorbidities, we then assessed the $\mathrm{CHA}_{2} \mathrm{DS}_{2}$-VASc score (Supplementary Tables S2, S3), the modified HAS-BLED score (Supplementary Tables S2, S4) (Lip et al., 2010; Friberg et al., 2012; Pisters et al., 2010). and the Charlson Comorbidity Index(Deyo et al., 1992; D'Hoore et al., 1996). A frailty score (based on an appropriate risk assessment index for the elderly) was evaluated for the two years preceding cohort entry (Crane et al., 2010; Fillion et al., 2019). Lastly, we assessed the prescriptions filled for several medications in the 2 weeks preceding cohort entry. Although data on aspirin claims were recorded, possible over-the-counter purchases might have made this variable less reliable.

\section{Statistical Analyses}

We used descriptive statistics to summarize the patients' demographic and clinical characteristics as a function of the DOAC initially prescribed after discharge from hospital.

In order to balance the distribution of baseline patient characteristics between groups, an inverse probability of treatment weighting (IPTW) method was employed (Austin and Stuart, 2015; Allan et al., 2020). We created IPTW populations for the following contrasts: 1) low-dose dabigatran vs. warfarin; 2) low-dose rivaroxaban vs. warfarin; 3) low-dose apixaban vs. warfarin; 4) low-dose dabigatran vs. apixaban; 5) low-dose rivaroxaban vs. apixaban; 6) low-dose dabigatran vs. rivaroxaban. We used a multivariable logistic regression model to estimate the propensity score defined as the probability of being in the observed (actual) treatment group, conditional on all baseline covariates. The IPTW weights used the inverse of the propensity score. This weighting creates a pseudo-population in which there is balance across treatment groups with respect to covariates included in the model (Supplementary Table S5). The IPTW approach attempts to minimize the impact of confounding bias in observational studies by approximating a randomization process used in randomized clinical trials. All weights were stabilized by multiplying by the marginal probability of being in the treatment group.

Descriptive statistics were used to characterize the patients after weighting by IPTW. We estimated standardized differences in baseline characteristics between the treatment groups, where differences $>10 \%$ may suggest meaningful imbalance (Austin and Stuart, 2015). For descriptive analyses, we presented the preand post-weighted between-group comparisons. We reported the outcomes per 100 person-years for each treatment in each IPTW population.

Patients were followed from the index date until the earliest of the following events: outcome, being institutionalized or hospitalized for more than 15 days, discontinuation of treatment, or switching to another oral anticoagulant or to another dosage, end of study, or death, whichever came first. The censoring was handled by the Cox proportional hazards model. We contrasted each low-dose DOAC with both warfarin and each other low-dose as references using IPTW to estimate marginal Cox hazard ratios (HRs) for outcomes under treatment (UT). We constructed confidence intervals using the validated robust standard error.

\section{Sensitivity Analyses}

We performed several sensitivity analyses of the effectiveness and safety composite outcomes for low-dose DOACs, relative to warfarin or to each other (Fralick et al., 2020). Firstly, we performed an intentto-treat (ITT) analysis in which we removed the censoring criteria of drug discontinuation or switching, so that all patients were followed up for 365 days unless they were censored for another reason. Secondly, we calculated an E-value as a guide to the potential impact of unmeasured confounding (VanderWeele and Ding, 2017). The E-value indicates how strongly an unmeasured confounder should be associated with use of each low-dose DOAC (relative to warfarin or another DOAC) to change the observed effects on effectiveness or safety to null, depending on the measured covariates. Lastly, we assessed the risk of diabetes complications (primary code of hospitalization (ICD-9: 250.1-250.9, 357.2, 366.41; ICD-10: E10-E14 excluding E10.9, E11.9, E12.9, E13.0, E14.9) and pneumonia (ICD9 code: 480-488 ICD10: J09-J18) as negative control outcomes. And, we assessed the impact of temporal trends accounted in the analysis by including the date of cohort entry in the IPTW matching. All statistical analyses were performed using SAS software (version 9.4, SAS Institute Inc. Cary, NC, United States). 


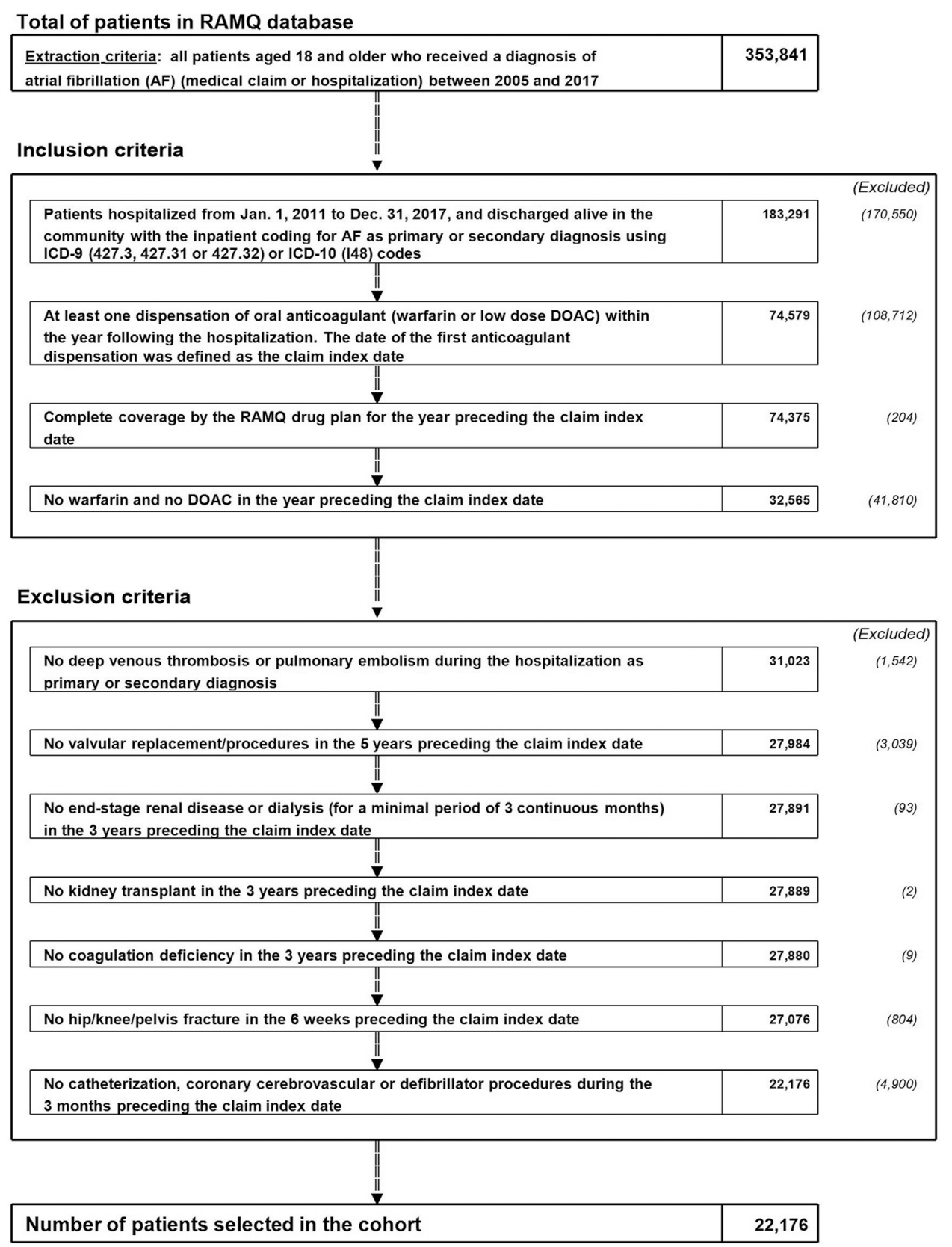

FIGURE 1 | Study flow chart. AF: atrial fibrillation; OAC: oral anticoagulant; RAMQ: Régie d'Assurance Maladie du Québec (Quebec administrative databases).

\section{RESULTS}

\section{Demographics and Clinical Characteristics of the Study Population}

A total of 22,176 patients with a confirmed diagnosis of AF received dabigatran $(n=1,929)$, rivaroxaban $(n=1,718)$, apixaban $(n=3,829)$ or warfarin $(n=14,700)$ (Figure 1). The characteristics of the study population for each DOAC after IPTW vs. warfarin are summarized in Table 1. In these groups, the mean age ranged from 80.2 to 82.2 , and $55.8-58.9 \%$ were women. The characteristics of the study population for each DOAC after IPTW vs. the other DOACs are summarized in Table 2. In these groups, the mean age ranged from 82.0 to 85.3 , and $59.6-66.1 \%$ were women. As shown in Supplementary Tables S5.1-S5.6, the absolute standardized differences in the IPTW populations were adequate.

\section{Cumulative Incidence Rates}

The annualized rates $[95 \%$ confidence interval (CI)] for effectiveness and safety outcomes when comparing low-dose DOAC vs. warfarin in as-treated and intent-to-treat analyses after IPTW are shown in Supplementary Tables S6.1, S6.2. 
TABLE 1 | Demographic and clinical characteristics of OAC users from 2011 to 2018, after IPTW (DOACs vs. warfarin).

\begin{tabular}{|c|c|c|c|c|c|c|}
\hline & \multicolumn{2}{|c|}{$\begin{array}{l}\text { IPTW dabigatran and warfarin } \\
\text { populations }\end{array}$} & \multicolumn{2}{|c|}{$\begin{array}{l}\text { IPTW rivaroxaban and warfarin } \\
\text { populations }\end{array}$} & \multicolumn{2}{|c|}{$\begin{array}{c}\text { IPTW apixaban and warfarin } \\
\text { populations }\end{array}$} \\
\hline & $\begin{array}{l}\text { Dabigatran } \\
110 \mathrm{mg} \\
\text { twice daily } \\
(N=1,929)\end{array}$ & $\begin{array}{c}\text { Warfarin } \\
(n= \\
14,700)\end{array}$ & $\begin{array}{c}\text { Rivaroxaban } \\
15 \mathrm{mg} \\
\text { once daily } \\
(N=1,718)\end{array}$ & $\begin{array}{c}\text { Warfarin } \\
(n= \\
14,700)\end{array}$ & $\begin{array}{c}\text { Apixaban } \\
2.5 \text { mg } \\
\text { twice daily } \\
\text { (n=3,829) }\end{array}$ & $\begin{array}{c}\text { Warfarin } \\
(n= \\
14,700)\end{array}$ \\
\hline Age, years, mean $\pm S D$ & $80.2(7.7)$ & $80.2(9.1)$ & $80.7 \pm 7.8$ & $80.4 \pm 9.1$ & $82.2 \pm 7.9$ & $81.5 \pm 9.1$ \\
\hline Females (\%) & $56.8 \%$ & $55.8 \%$ & $57.0 \%$ & $56.1 \%$ & $58.9 \%$ & $58.2 \%$ \\
\hline Pampalon index: elevated social deprivation & $26.7 \%$ & $26.6 \%$ & $26.5 \%$ & $26.6 \%$ & $26.6 \%$ & $26.6 \%$ \\
\hline Pampalon index: elevated material deprivation & $25.7 \%$ & $25.9 \%$ & $25.6 \%$ & $25.9 \%$ & $25.7 \%$ & $25.9 \%$ \\
\hline $\mathrm{CHA}_{2} \mathrm{DS}_{2}$-VASc score $(\text { mean } \pm \mathrm{SD})^{\star}$ & $4.0 \pm 1.3$ & $3.9 \pm 1.4$ & $4.0 \pm 1.3$ & $4.0 \pm 1.4$ & $4.2 \pm 1.3$ & $4.0 \pm 1.4$ \\
\hline $\mathrm{CHA}_{2} \mathrm{DS}_{2}$-VASc score $0-1$ & $2.6 \%$ & $3.9 \%$ & $2.6 \%$ & $3.7 \%$ & $1.5 \%$ & $3.2 \%$ \\
\hline $\mathrm{CHA}_{2} \mathrm{DS}_{2}-\mathrm{VASc}$ score 2-3 & $32.8 \%$ & $31.9 \%$ & $30.7 \%$ & $31.5 \%$ & $28.2 \%$ & $29.8 \%$ \\
\hline $\mathrm{CHA}_{2} \mathrm{DS}_{2}$-VASc score 4 & $32.5 \%$ & $31.1 \%$ & $33.5 \%$ & $31.3 \%$ & $33.2 \%$ & $31.9 \%$ \\
\hline $\mathrm{CHA}_{2} \mathrm{DS}_{2}$-VASc score $\geq 5$ & $32.1 \%$ & $33.1 \%$ & $33.2 \%$ & $33.5 \%$ & $37.1 \%$ & $35.1 \%$ \\
\hline HAS-BLED score $(\text { mean } \pm S D)^{\star}$ & $3.3 \pm 1.2$ & $3.0 \pm 1.3$ & $3.4 \pm 1.3$ & $3.3 \pm 1.3$ & $3.4 \pm 1.3$ & $3.3 \pm 1.3$ \\
\hline HAS-BLED score <3 & $25.7 \%$ & $27.4 \%$ & $25.9 \%$ & $26.8 \%$ & $23.9 \%$ & $26.6 \%$ \\
\hline HAS-BLED score $\geq 3$ & $74.3 \%$ & $72.6 \%$ & $74.1 \%$ & $73.2 \%$ & $76.1 \%$ & $73.4 \%$ \\
\hline \multicolumn{7}{|l|}{ Charlson comorbidity index* } \\
\hline Charlson comorbidity index (mean $\pm \mathrm{SD}$ ) & $4.9 \pm 3.5$ & $4.9 \pm 3.4$ & $5.2 \pm 3.7$ & $5.0 \pm 3.4$ & $5.3 \pm 3.5$ & $5.0 \pm 3.4$ \\
\hline Charlson comorbidity index (median [IQR]) & $4.0(2.0-7.0)$ & $4.0(2.0-7.0)$ & $5.0(3.0-7.0)$ & $4.0(2.0-7.0)$ & $5.0(3.0-7.0)$ & $4.0(2.0-7.0)$ \\
\hline Charlson comorbidity index $<4$ & $40.9 \%$ & $39.1 \%$ & $36.6 \%$ & $38.3 \%$ & $34.0 \%$ & $38.2 \%$ \\
\hline Charlson comorbidity index $\geq 4$ & $59.1 \%$ & $60.9 \%$ & $63.4 \%$ & $61.7 \%$ & $66.0 \%$ & $61.8 \%$ \\
\hline Frailty score (mean \pm SD) & $12.7 \pm 6.9$ & $12.6 \pm 7.0$ & $12.9 \pm 6.9$ & $12.6 \pm 7.0$ & $13.3 \pm 6.9$ & $12.9 \pm 7.1$ \\
\hline Robust (frailty score $\leq-1$ ) & $0 \%$ & $0 \%$ & $0 \%$ & $0 \%$ & $0 \%$ & $0 \%$ \\
\hline Well (frailty score: 0-3) & $6.7 \%$ & $8.0 \%$ & $6.9 \%$ & $7.9 \%$ & $5.5 \%$ & $7.2 \%$ \\
\hline Well/comorbidities (frailty score: 4-8) & $24.2 \%$ & $25.4 \%$ & $23.7 \%$ & $25.2 \%$ & $24.6 \%$ & $24.6 \%$ \\
\hline Pre-frail (frailty score: 9-15) & $35.2 \%$ & $33.0 \%$ & $35.6 \%$ & $33.1 \%$ & $33.6 \%$ & $33.5 \%$ \\
\hline Frail (frailty score: $\geq 16$ ) & $33.9 \%$ & $33.6 \%$ & $33.8 \%$ & $33.8 \%$ & $36.3 \%$ & $34.7 \%$ \\
\hline Hypertension & $84.4 \%$ & $84.6 \%$ & $86.0 \%$ & $84.8 \%$ & $86.1 \%$ & $84.7 \%$ \\
\hline Coronary artery disease & $59.3 \%$ & $59.1 \%$ & $60.3 \%$ & $59.4 \%$ & $59.8 \%$ & $58.8 \%$ \\
\hline Acute myocardial infarction & $14.0 \%$ & $15.0 \%$ & $16.5 \%$ & $15.6 \%$ & $17.1 \%$ & $15.9 \%$ \\
\hline Chronic heart failure & $41.2 \%$ & $43.1 \%$ & $44.5 \%$ & $43.6 \%$ & $46.1 \%$ & $44.0 \%$ \\
\hline Cardiomyopathy & $5.6 \%$ & $6.2 \%$ & $6.2 \%$ & $6.3 \%$ & $5.7 \%$ & $6.1 \%$ \\
\hline Other cardiac rhythm disorders & $20.3 \%$ & $20.7 \%$ & $20.2 \%$ & $20.2 \%$ & $19.7 \%$ & $20.1 \%$ \\
\hline Valvular heart disease & $22.3 \%$ & $22.3 \%$ & $22.0 \%$ & $22.6 \%$ & $23.2 \%$ & $22.8 \%$ \\
\hline Stroke/Transient ischemic attack & $21.5 \%$ & $21.4 \%$ & $20.7 \%$ & $20.9 \%$ & $22.1 \%$ & $20.8 \%$ \\
\hline Peripheral vascular (arterial) disease & $23.3 \%$ & $24.4 \%$ & $25.6 \%$ & $24.7 \%$ & $25.9 \%$ & $24.4 \%$ \\
\hline Dyslipidemia & $51.8 \%$ & $53.4 \%$ & $53.2 \%$ & $53.5 \%$ & $53.2 \%$ & $52.7 \%$ \\
\hline Diabetes & $35.0 \%$ & $37.9 \%$ & $38.8 \%$ & $38.0 \%$ & $37.7 \%$ & $36.8 \%$ \\
\hline Major bleeding & $31.8 \%$ & $32.4 \%$ & $34.8 \%$ & $32.8 \%$ & $36.1 \%$ & $33.1 \%$ \\
\hline Major intracranial bleeding & $3.6 \%$ & $3.4 \%$ & $4.4 \%$ & $3.4 \%$ & $5.1 \%$ & $3.4 \%$ \\
\hline Major gastrointestinal bleeding & $8.9 \%$ & $8.1 \%$ & $8.4 \%$ & $8.2 \%$ & $9.3 \%$ & $8.1 \%$ \\
\hline Major bleeding at other sites & $24.3 \%$ & $25.4 \%$ & $27.2 \%$ & $25.9 \%$ & $27.4 \%$ & $26.4 \%$ \\
\hline Chronic renal failure & $42.6 \%$ & $43.3 \%$ & $49.0 \%$ & $45.1 \%$ & $51.3 \%$ & $45.9 \%$ \\
\hline
\end{tabular}


TABLE 1 | (Continued) Demographic and clinical characteristics of OAC users from 2011 to 2018, after IPTW (DOACs vs. warfarin).

\begin{tabular}{|c|c|c|c|c|c|c|}
\hline & \multicolumn{2}{|c|}{$\begin{array}{l}\text { IPTW dabigatran and warfarin } \\
\text { populations }\end{array}$} & \multicolumn{2}{|c|}{$\begin{array}{l}\text { IPTW rivaroxaban and warfarin } \\
\text { populations }\end{array}$} & \multicolumn{2}{|c|}{$\begin{array}{c}\text { IPTW apixaban and warfarin } \\
\text { populations }\end{array}$} \\
\hline & $\begin{array}{l}\text { Dabigatran } \\
110 \mathrm{mg} \\
\text { twice daily } \\
(N=1,929)\end{array}$ & $\begin{array}{c}\text { Warfarin } \\
(n= \\
14,700)\end{array}$ & $\begin{array}{c}\text { Rivaroxaban } \\
15 \mathrm{mg} \\
\text { once daily } \\
(N=1,718)\end{array}$ & $\begin{array}{c}\text { Warfarin } \\
(n= \\
14,700)\end{array}$ & $\begin{array}{c}\text { Apixaban } \\
2.5 \text { mg } \\
\text { twice daily } \\
\text { (n = 3,829) }\end{array}$ & $\begin{array}{c}\text { Warfarin } \\
(n= \\
14,700)\end{array}$ \\
\hline Chronic renal failure $\leq 30 \mathrm{ml} / \mathrm{min}$ & $5.7 \%$ & $7.4 \%$ & $7.2 \%$ & $7.6 \%$ & $8.6 \%$ & $7.2 \%$ \\
\hline Acute renal failure & $25.1 \%$ & $27.6 \%$ & $30.9 \%$ & $29.1 \%$ & $33.6 \%$ & $29.5 \%$ \\
\hline Liver disease & $2.2 \%$ & $2.2 \%$ & $2.6 \%$ & $2.2 \%$ & $2.4 \%$ & $2.1 \%$ \\
\hline Chronic obstructive pulmonary disease/asthma & $37.6 \%$ & $38.5 \%$ & $40.5 \%$ & $38.8 \%$ & $37.6 \%$ & $37.9 \%$ \\
\hline Helicobacter pylori infection & $0.8 \%$ & $0.8 \%$ & $1.5 \%$ & $0.8 \%$ & $0.6 \%$ & $0.8 \%$ \\
\hline Depression & $11.8 \%$ & $11.5 \%$ & $11.0 \%$ & $11.4 \%$ & $11.3 \%$ & $11.5 \%$ \\
\hline \multicolumn{7}{|l|}{ Medical procedures* } \\
\hline Cardiac catheterization & $3.5 \%$ & $3.8 \%$ & $3.9 \%$ & $3.8 \%$ & $3.8 \%$ & $3.7 \%$ \\
\hline Percutaneous coronary intervention-stent & $3.6 \%$ & $2.9 \%$ & $3.3 \%$ & $3.0 \%$ & $2.9 \%$ & $2.8 \%$ \\
\hline Coronary artery bypass grafting & $1.0 \%$ & $0.7 \%$ & $0.9 \%$ & $0.7 \%$ & $0.5 \%$ & $0.6 \%$ \\
\hline Medical procedures for cerebrovascular disease & $1.0 \%$ & $1.1 \%$ & $1.3 \%$ & $1.1 \%$ & $1.3 \%$ & $1.0 \%$ \\
\hline Medical procedures for a defibrillator & $0.8 \%$ & $0.5 \%$ & $0.1 \%$ & $0.4 \%$ & $0.0 \%$ & $0.4 \%$ \\
\hline \multicolumn{7}{|l|}{ Medications (2 weeks prior cohort entry) } \\
\hline Statin & $46.8 \%$ & $47.4 \%$ & $47.5 \%$ & $47.3 \%$ & $46.0 \%$ & $46.4 \%$ \\
\hline Antiplatelet agents (excluding low-dose ASA) & $6.2 \%$ & $6.0 \%$ & $6.3 \%$ & $6.1 \%$ & $6.2 \%$ & $6.1 \%$ \\
\hline Low-dose ASA & $31.8 \%$ & $31.5 \%$ & $31.3 \%$ & $31.4 \%$ & $30.9 \%$ & $30.8 \%$ \\
\hline Proton pump inhibitors & $46.1 \%$ & $45.8 \%$ & $46.2 \%$ & $45.7 \%$ & $47.0 \%$ & $45.7 \%$ \\
\hline NSAIDs & $1.4 \%$ & $1.4 \%$ & $1.3 \%$ & $1.3 \%$ & $1.3 \%$ & $1.3 \%$ \\
\hline Digoxin & $14.6 \%$ & $13.5 \%$ & $12.9 \%$ & $13.3 \%$ & $12.3 \%$ & $12.8 \%$ \\
\hline Amiodarone or propafenone & $9.9 \%$ & $10.1 \%$ & $10.4 \%$ & $10.1 \%$ & $9.7 \%$ & $10.1 \%$ \\
\hline Antidepressants & $9.0 \%$ & $8.7 \%$ & $8.5 \%$ & $8.7 \%$ & $8.5 \%$ & $8.8 \%$ \\
\hline B-blockers & $60.8 \%$ & $62.2 \%$ & $62.2 \%$ & $62.4 \%$ & $61.2 \%$ & $62.9 \%$ \\
\hline Calcium channel blockers & $39.1 \%$ & $39.6 \%$ & $39.7 \%$ & $39.8 \%$ & $40.8 \%$ & $39.9 \%$ \\
\hline Renin-angiotensin system inhibitors & $38.9 \%$ & $38.2 \%$ & $38.3 \%$ & $37.8 \%$ & $37.3 \%$ & $37.3 \%$ \\
\hline Diuretics & $42.3 \%$ & $43.4 \%$ & $45.6 \%$ & $44.1 \%$ & $46.1 \%$ & $44.2 \%$ \\
\hline Loop diuretics & $35.2 \%$ & $36.2 \%$ & $38.6 \%$ & $36.8 \%$ & $39.2 \%$ & $37.3 \%$ \\
\hline Antidiabetics & $20.8 \%$ & $22.4 \%$ & $23.4 \%$ & $22.5 \%$ & $21.9 \%$ & $21.7 \%$ \\
\hline PGP inhibitor use ${ }^{\ddagger}$ & $61.0 \%$ & $61.6 \%$ & $61.9 \%$ & $61.9 \%$ & $62.1 \%$ & $61.7 \%$ \\
\hline \multicolumn{7}{|l|}{ Medical services (in the year prior to entry,\%) } \\
\hline Number of visits to a specialist (mean \pm SD) & $1.3 \pm 2.2$ & $1.2 \pm 2.3$ & $1.2 \pm 2.0$ & $1.2 \pm 2.1$ & $1.2 \pm 2.2$ & $1.2 \pm 2.4$ \\
\hline Number of family physician visits (mean $\pm \mathrm{SD}$ ) & $1.3 \pm 3.0$ & $1.3 \pm 3.0$ & $1.3 \pm 2.9$ & $1.3 \pm 3.0$ & $1.3 \pm 2.8$ & $1.3 \pm 3.0$ \\
\hline Number of emergency room visits (mean \pm SD) & $3.3 \pm 2.8$ & $3.2 \pm 2.8$ & $3.3 \pm 2.6$ & $3.2 \pm 2.8$ & $3.3 \pm 2.6$ & $3.2 \pm 2.8$ \\
\hline \multicolumn{7}{|l|}{ Hospital services (in the 3 years prior to entry,\%) } \\
\hline$\geq 2$ all-cause hospital admissions & $61.8 \%$ & $58.3 \%$ & $59.3 \%$ & $58.2 \%$ & $57.4 \%$ & $58.0 \%$ \\
\hline $\begin{array}{l}\text { Number of all-cause hospital admissions (mean } \\
\text { admission }( \pm \mathrm{SD})\end{array}$ & $2.4 \pm 1.7$ & $2.4 \pm 1.8$ & $2.4 \pm 2.0$ & $2.4 \pm 1.8$ & $2.4 \pm 1.9$ & $2.4 \pm 1.9$ \\
\hline Hospital length of stay (mean $\pm \mathrm{SD}$ ) & $11.1 \pm 14.2$ & $10.8 \pm 12.0$ & $11.1 \pm 13.4$ & $10.8 \pm 12.0$ & $11.2 \pm 13.2$ & $11.2 \pm 13.2$ \\
\hline
\end{tabular}

${ }^{*}$ In the 3 years to the cohort entry; ${ }^{\ddagger} P$-glycoprotein. IPTW: inverse probability of treatment weighting; ${ }^{\dagger}$ Antidepressants: SSRIs (citalopram, escitalopram, fluoxetine, paroxetine, sertraline) 
TABLE 2 | Demographic and clinical characteristics of OACs users from 2011 to 2018, after IPTW (comparisons of DOACs).

\begin{tabular}{|c|c|c|c|c|c|c|}
\hline & \multicolumn{2}{|c|}{$\begin{array}{l}\text { IPTW dabigatran and apixaban } \\
\text { populations }\end{array}$} & \multicolumn{2}{|c|}{$\begin{array}{l}\text { IPTW rivaroxaban and apixaban } \\
\text { populations }\end{array}$} & \multicolumn{2}{|c|}{$\begin{array}{l}\text { IPTW dabigatran and rivaroxabar } \\
\text { populations }\end{array}$} \\
\hline & $\begin{array}{l}\text { Dabigatran } \\
110 \mathrm{mg} \\
\text { twice daily } \\
(N=1,929)\end{array}$ & $\begin{array}{c}\text { Apixaban } \\
2.5 \mathrm{mg} \\
\text { twice daily } \\
\text { (n=3,829) }\end{array}$ & $\begin{array}{c}\text { Rivaroxaban } \\
15 \mathrm{mg} \\
\text { once daily } \\
(N=1,718)\end{array}$ & $\begin{array}{c}\text { Apixaban } \\
2.5 \mathrm{mg} \\
\text { twice daily } \\
\text { (n=3,829) }\end{array}$ & $\begin{array}{c}\text { Dabigatran } \\
110 \mathrm{mg} \\
\text { twice daily } \\
(N=1,929)\end{array}$ & $\begin{array}{c}\text { Rivaroxaban } \\
15 \mathrm{mg} \\
\text { once daily } \\
(N=1,718)\end{array}$ \\
\hline Age-mean $\pm S D$ & $84.2 \pm 6.6$ & $84.2 \pm 7.8$ & $85.3 \pm 6.7$ & $85.2 \pm 7.0$ & $81.9 \pm 7.0$ & $82.0 \pm 7.5$ \\
\hline Female (\%) & $64.5 \%$ & $64.9 \%$ & $65.8 \%$ & $66.1 \%$ & $59.6 \%$ & $59.8 \%$ \\
\hline Pampalon index elevated social deprivation & $26.6 \%$ & $26.6 \%$ & $26.5 \%$ & $26.6 \%$ & $26.6 \%$ & $26.5 \%$ \\
\hline Pampalon index elevated material deprivation & $25.7 \%$ & $25.7 \%$ & $25.6 \%$ & $25.7 \%$ & $25.7 \%$ & $25.6 \%$ \\
\hline $\mathrm{CHA}_{2} \mathrm{DS}_{2}$-VASc score (mean $\left.\pm \mathrm{SD}\right)^{*}$ & $4.1 \pm 1.2$ & $4.1 \pm 1.3$ & $4.2 \pm 1.2$ & $4.2 \pm 1.2$ & $3.9 \pm 1.2$ & $3.9 \pm 1.3$ \\
\hline $\mathrm{CHA}_{2} \mathrm{DS}_{2}$-VASc score $0-1$ & $0.9 \%$ & $1.4 \%$ & $0.8 \%$ & $0.9 \%$ & $1.7 \%$ & $2.7 \%$ \\
\hline $\mathrm{CHA}_{2} \mathrm{DS}_{2}$-VASc score $2-3$ & $28.5 \%$ & $28.4 \%$ & $25.8 \%$ & $26.1 \%$ & $34.5 \%$ & $33.3 \%$ \\
\hline $\mathrm{CHA}_{2} \mathrm{DS}_{2}$-VASc score 4 & $36.4 \%$ & $35.1 \%$ & $37.4 \%$ & $36.1 \%$ & $35.5 \%$ & $34.8 \%$ \\
\hline $\mathrm{CHA}_{2} \mathrm{DS}_{2}$-VASc score $\geq 5$ & $34.2 \%$ & $35.1 \%$ & $36.0 \%$ & $36.9 \%$ & $28.3 \%$ & $29.2 \%$ \\
\hline HAS-BLED score $(\text { mean } \pm \mathrm{SD})^{\star}$ & $3.2 \pm 1.2$ & $3.2 \pm 1.3$ & $3.2 \pm 1.3$ & $3.2 \pm 1.3$ & $3.0 \pm 1.2$ & $3.1 \pm 1.3$ \\
\hline HAS-BLED score <3 & $28.7 \%$ & $31.1 \%$ & $29.4 \%$ & $28.9 \%$ & $31.9 \%$ & $34.0 \%$ \\
\hline HAS-BLED score $\geq 3$ & $71.3 \%$ & $68.9 \%$ & $70.6 \%$ & $71.1 \%$ & $68.1 \%$ & $66.0 \%$ \\
\hline \multicolumn{7}{|l|}{ Charlson score ${ }^{\star}$} \\
\hline Charlson comorbidity index (mean \pm SD) & $4.6 \pm 3.4$ & $4.6 \pm 3.3$ & $4.8 \pm 3.4$ & $4.7 \pm 3.4$ & $4.4 \pm 3.4$ & $4.4 \pm 3.4$ \\
\hline Charlson comorbidity index (median [IQR]) & $4.0(2.0-6.0)$ & $4.0(2.0-6.0)$ & $4.0(2.0-6.0)$ & $4.0(2.0-6.0)$ & $4.0(2.0-6.0)$ & $4.0(2.0-6.0)$ \\
\hline Charlson comorbidity index $<4$ & $45.0 \%$ & $43.2 \%$ & $41.7 \%$ & $41.2 \%$ & $47.8 \%$ & $48.0 \%$ \\
\hline Charlson comorbidity index $\geq 4$ & $55.0 \%$ & $56.8 \%$ & $58.3 \%$ & $58.8 \%$ & $52.2 \%$ & $52.0 \%$ \\
\hline Frailty score (mean \pm SD) & $13.1 \pm 6.8$ & $13.0 \pm 7.0$ & $13.3 \pm 6.8$ & $13.2 \pm 6.9$ & $12.2 \pm 6.8$ & $12.2 \pm 6.7$ \\
\hline Robust (frailty score $\leq-1$ ) & $0 \%$ & $0 \%$ & $0 \%$ & $0 \%$ & $0 \%$ & $0 \%$ \\
\hline Well (frailty score: 0-3) & $6.3 \%$ & $6.3 \%$ & $5.3 \%$ & $5.5 \%$ & $8.0 \%$ & $8.1 \%$ \\
\hline Well/comorbidities (frailty score: 4-8) & $23.1 \%$ & $25.0 \%$ & $22.8 \%$ & $24.1 \%$ & $26.1 \%$ & $26.3 \%$ \\
\hline Pre-frail (frailty score: 9-15) & $35.0 \%$ & $34.9 \%$ & $37.9 \%$ & $35.8 \%$ & $35.2 \%$ & $36.1 \%$ \\
\hline Frail (frailty score: $\geq 16$ ) & $35.6 \%$ & $33.8 \%$ & $34.0 \%$ & $34.6 \%$ & $30.7 \%$ & $29.5 \%$ \\
\hline Hypertension & $83.4 \%$ & $83.1 \%$ & $83.7 \%$ & $83.9 \%$ & $83.2 \%$ & $82.9 \%$ \\
\hline Coronary artery disease & $52.4 \%$ & $52.7 \%$ & $53.2 \%$ & $53.4 \%$ & $52.5 \%$ & $52.5 \%$ \\
\hline Acute myocardial infarction & $13.9 \%$ & $14.4 \%$ & $15.8 \%$ & $15.8 \%$ & $11.9 \%$ & $12.2 \%$ \\
\hline Chronic heart failure & $40.2 \%$ & $40.4 \%$ & $42.1 \%$ & $41.7 \%$ & $36.7 \%$ & $36.8 \%$ \\
\hline Cardiomyopathy & $4.5 \%$ & $4.9 \%$ & $5.0 \%$ & $5.2 \%$ & $5.0 \%$ & $4.9 \%$ \\
\hline Other cardiac rhythm disorders & $20.1 \%$ & $19.9 \%$ & $18.3 \%$ & $18.5 \%$ & $20.7 \%$ & $20.5 \%$ \\
\hline Valvular heart disease & $20.6 \%$ & $20.5 \%$ & $21.1 \%$ & $21.2 \%$ & $18.2 \%$ & $18.2 \%$ \\
\hline Stroke/Transient ischemic attack & $21.6 \%$ & $20.5 \%$ & $19.6 \%$ & $19.2 \%$ & $20.7 \%$ & $20.4 \%$ \\
\hline Peripheral vascular (arterial) disease & $20.3 \%$ & $20.2 \%$ & $21.0 \%$ & $21.2 \%$ & $20.2 \%$ & $20.5 \%$ \\
\hline Dyslipidemia & $47.9 \%$ & $49.2 \%$ & $49.3 \%$ & $49.6 \%$ & $50.0 \%$ & $49.7 \%$ \\
\hline Diabetes & $28.7 \%$ & $29.4 \%$ & $30.0 \%$ & $29.7 \%$ & $30.5 \%$ & $29.9 \%$ \\
\hline Major bleeding & $33.5 \%$ & $32.3 \%$ & $33.1 \%$ & $33.1 \%$ & $29.3 \%$ & $29.0 \%$ \\
\hline Major intracranial bleeding & $3.3 \%$ & $5.0 \%$ & $4.0 \%$ & $4.6 \%$ & $3.2 \%$ & $3.7 \%$ \\
\hline Major gastrointestinal bleeding & $9.1 \%$ & $7.9 \%$ & $7.3 \%$ & $8.2 \%$ & $8.7 \%$ & $7.1 \%$ \\
\hline Major bleeding at other sites & $26.0 \%$ & $24.1 \%$ & $26.4 \%$ & $25.2 \%$ & $21.9 \%$ & $22.3 \%$ \\
\hline Chronic renal failure & $39.3 \%$ & $38.3 \%$ & $43.3 \%$ & $43.5 \%$ & $32.7 \%$ & $32.8 \%$ \\
\hline
\end{tabular}

(Continued on following page) 
TABLE 2 | (Continued) Demographic and clinical characteristics of OACs users from 2011 to 2018, after IPTW (comparisons of DOACs).

\begin{tabular}{|c|c|c|c|c|c|c|}
\hline & \multicolumn{2}{|c|}{$\begin{array}{l}\text { IPTW dabigatran and apixaban } \\
\text { populations }\end{array}$} & \multicolumn{2}{|c|}{$\begin{array}{l}\text { IPTW rivaroxaban and apixaban } \\
\text { populations }\end{array}$} & \multicolumn{2}{|c|}{$\begin{array}{l}\text { IPTW dabigatran and rivaroxaban } \\
\text { populations }\end{array}$} \\
\hline & $\begin{array}{c}\text { Dabigatran } \\
110 \mathrm{mg} \\
\text { twice daily } \\
(N=1,929)\end{array}$ & $\begin{array}{c}\text { Apixaban } \\
2.5 \mathrm{mg} \\
\text { twice daily } \\
\text { ( } n=3,829)\end{array}$ & $\begin{array}{c}\text { Rivaroxaban } \\
15 \mathrm{mg} \\
\text { once daily } \\
(N=1,718)\end{array}$ & $\begin{array}{c}\text { Apixaban } \\
2.5 \mathrm{mg} \\
\text { twice daily } \\
\text { ( } n=3,829 \text { ) }\end{array}$ & $\begin{array}{l}\text { Dabigatran } \\
110 \text { mg } \\
\text { twice daily } \\
(N=1,929)\end{array}$ & $\begin{array}{c}\text { Rivaroxaban } \\
15 \mathrm{mg} \\
\text { once daily } \\
(N=1,718)\end{array}$ \\
\hline Chronic renal failure $\leq 30 \mathrm{ml} / \mathrm{min}$ & $3.4 \%$ & $2.8 \%$ & $3.7 \%$ & $3.4 \%$ & $2.1 \%$ & $2.2 \%$ \\
\hline Acute renal failure & $23.5 \%$ & $23.0 \%$ & $27.0 \%$ & $27.1 \%$ & $18.6 \%$ & $18.7 \%$ \\
\hline Liver disease & $1.8 \%$ & $1.8 \%$ & $1.5 \%$ & $1.7 \%$ & $2.0 \%$ & $1.9 \%$ \\
\hline Chronic obstructive pulmonary disease/asthma & $35.1 \%$ & $34.4 \%$ & $35.0 \%$ & $35.2 \%$ & $36.3 \%$ & $36.1 \%$ \\
\hline Helicobacter pylori infection & $0.8 \%$ & $0.6 \%$ & $1.2 \%$ & $0.6 \%$ & $0.7 \%$ & $1.2 \%$ \\
\hline Depression & $12.6 \%$ & $12.8 \%$ & $12.3 \%$ & $12.4 \%$ & $13.1 \%$ & $13.1 \%$ \\
\hline \multicolumn{7}{|l|}{ Medical procedures* } \\
\hline Cardiac catheterization & $2.9 \%$ & $2.7 \%$ & $2.7 \%$ & $2.6 \%$ & $2.9 \%$ & $2.8 \%$ \\
\hline Percutaneous coronary intervention-stent & $2.3 \%$ & $2.3 \%$ & $2.5 \%$ & $2.4 \%$ & $2.4 \%$ & $2.6 \%$ \\
\hline Coronary artery bypass grafting & $0.3 \%$ & $0.2 \%$ & $0.3 \%$ & $0.3 \%$ & $0.6 \%$ & $0.6 \%$ \\
\hline $\begin{array}{l}\text { Medical procedures for cerebrovascular } \\
\text { disease }\end{array}$ & $1.0 \%$ & $0.7 \%$ & $0.7 \%$ & $0.7 \%$ & $0.9 \%$ & $0.9 \%$ \\
\hline Medical procedures for a defibrillator & $0.2 \%$ & $0.0 \%$ & $0.02 \%$ & $0.00 \%$ & $0.3 \%$ & $0.3 \%$ \\
\hline \multicolumn{7}{|l|}{ Medications (2 weeks prior to entry) } \\
\hline Statin & $41.0 \%$ & $41.9 \%$ & $40.8 \%$ & $41.3 \%$ & $42.8 \%$ & $43.0 \%$ \\
\hline Antiplatelets agents exclusing low-dose ASA) & $6.0 \%$ & $5.5 \%$ & $5.7 \%$ & $5.7 \%$ & $5.1 \%$ & $4.9 \%$ \\
\hline Low-dose ASA & $28.3 \%$ & $26.9 \%$ & $26.5 \%$ & $26.4 \%$ & $27.7 \%$ & $27.4 \%$ \\
\hline Proton pump inhibitors & $43.7 \%$ & $43.8 \%$ & $43.7 \%$ & $43.6 \%$ & $43.1 \%$ & $42.9 \%$ \\
\hline NSAIDs & $1.3 \%$ & $1.3 \%$ & $1.1 \%$ & $1.1 \%$ & $1.3 \%$ & $1.3 \%$ \\
\hline Digoxin & $11.9 \%$ & $11.6 \%$ & $11.1 \%$ & $10.9 \%$ & $12.9 \%$ & $12.9 \%$ \\
\hline Amiodarone or propafenone & $10.4 \%$ & $9.7 \%$ & $9.6 \%$ & $9.8 \%$ & $10.1 \%$ & $9.9 \%$ \\
\hline Antidepressants & $10.5 \%$ & $10.1 \%$ & $9.6 \%$ & $9.8 \%$ & $9.5 \%$ & $9.2 \%$ \\
\hline B-blockers & $63.3 \%$ & $63.4 \%$ & $65.1 \%$ & $64.7 \%$ & $62.9 \%$ & $63.1 \%$ \\
\hline Calcium channel blockers & $39.6 \%$ & $38.8 \%$ & $38.9 \%$ & $39.2 \%$ & $37.8 \%$ & $37.5 \%$ \\
\hline Renin-angiotensin system inhibitors & $38.4 \%$ & $37.0 \%$ & $35.3 \%$ & $35.6 \%$ & $39.2 \%$ & $39.3 \%$ \\
\hline Diuretics & $41.0 \%$ & $40.7 \%$ & $42.0 \%$ & $42.4 \%$ & $38.8 \%$ & $38.7 \%$ \\
\hline Loop diuretics & $34.4 \%$ & $34.1 \%$ & $35.1 \%$ & $35.5 \%$ & $30.8 \%$ & $30.8 \%$ \\
\hline Antidiabetics & $16.7 \%$ & $17.2 \%$ & $17.2 \%$ & $17.0 \%$ & $17.7 \%$ & $17.1 \%$ \\
\hline PGP inhibitor use ${ }^{\ddagger}$ & $59.4 \%$ & $59.8 \%$ & $60.5 \%$ & $60.4 \%$ & $59.5 \%$ & $59.3 \%$ \\
\hline \multicolumn{7}{|l|}{ Medical services ${ }^{\star}$} \\
\hline Number of visits to a specialist (mean \pm SD) & $1.4 \pm 2.9$ & $1.3 \pm 2.8$ & $1.3 \pm 2.5$ & $1.3 \pm 2.6$ & $1.3 \pm 2.6$ & $1.3 \pm 2.5$ \\
\hline Number of family physician visits (mean \pm SD) & $1.3 \pm 3.0$ & $1.3 \pm 3.0$ & $1.3 \pm 2.9$ & $1.3 \pm 3.0$ & $1.4 \pm 3.1$ & $1.4 \pm 3.0$ \\
\hline Number of emergency room visits (mean \pm SD) & $3.2 \pm 2.6$ & $3.3 \pm 2.7$ & $3.3 \pm 2.4$ & $3.3 \pm 2.6$ & $3.2 \pm 2.8$ & $3.2 \pm 2.5$ \\
\hline \multicolumn{7}{|l|}{ Hospital services (in the year before entry,\%) } \\
\hline$\geq 2$ all-cause hospital admissions & $59.2 \%$ & $54.8 \%$ & $56.5 \%$ & $54.1 \%$ & $58.3 \%$ & $57.4 \%$ \\
\hline $\begin{array}{l}\text { Number of all-cause hospital admissions (mean } \\
\text { admission }( \pm S D)\end{array}$ & $2.3 \pm 1.5$ & $2.3 \pm 1.7$ & $2.2 \pm 1.6$ & $2.2 \pm 1.7$ & $2.3 \pm 1.6$ & $2.3 \pm 1.7$ \\
\hline Hospital length of stay (mean \pm SD) & $10.1 \pm 10.9$ & $10.0 \pm 11.3$ & $10.3 \pm 11.5$ & $10.3 \pm 11.5$ & $9.4 \pm 11.0$ & $9.3 \pm 10.9$ \\
\hline
\end{tabular}

${ }^{*}$ In the 3 years to the cohort entry; ${ }^{\ddagger}$ P-glycoprotein. IPTW: inverse probability of treatment weighting; ${ }^{\dagger}$ Antidepressants: SSRIs (citalopram, escitalopram, fluoxetine, paroxetine, sertraline) 


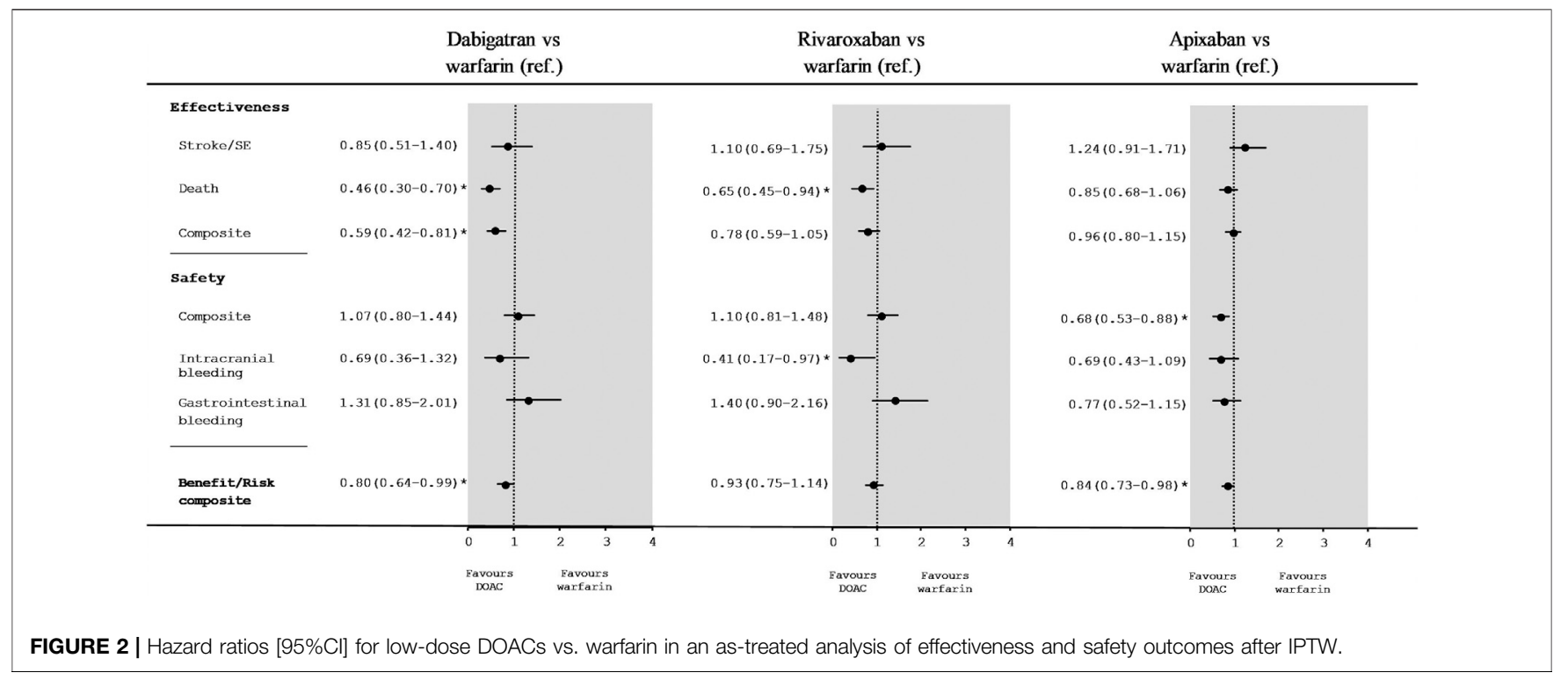

Similarly, the rates for low-dose DOACs vs. the other DOACs are shown in Supplementary Tables S6.3, S6.4.

\section{Effectiveness and Safety Outcomes of Direct Oral Anticoagulants vs. Warfarin}

Figure 2 shows the HRs [95\%CI] for the primary and secondary outcomes in IPTW populations taking low-dose DOACs vs. warfarin. The difference between dabigatran and warfarin was not statistically significant for the primary effectiveness outcome (stroke/SE) (HR [95\%CI]: $0.85[0.51-1.40]$ ) and the safety composite (1.07 [0.80-1.44]). The HR [95\%CI] for all-cause mortality was $0.45(0.30-0.70])$, the $\mathrm{HR}$ for the effectiveness composite was $0.59[0.42-0.81]$ and the HR benefit/risk composite was 0.80 [0.64-0.99]). Similarly, the difference between rivaroxaban and warfarin was not statistically significant for the primary outcome (1.10 [0.69-1.75]), the safety composite $(1.10[0.81-1.48])$ or the benefit/risk composite (0.93 [0.75-1.14]). The HR [95\%CI] for all-cause mortality was $0.65[0.45-0.94])$. Lastly, there were no significant differences between apixaban and warfarin with regard to the primary outcome (HR [95\%CI]: 1.24 [0.91-1.71]) but was significant for the safety composite $(0.68$ [0.53-0.88]) or the benefit/risk composite (0.84 [0.73-0.98]). The HR [95\%CI] for all-cause mortality $(0.85$ [0.68-1.06]) was not statistically significant.

\section{Effectiveness and Safety Outcomes When Comparing Direct Oral Anticoagulants With Each Other}

Figure 3 shows the HRs [95\%CI] for the effectiveness and safety outcomes in IPTW populations taking one low-dose DOAC vs. another low-dose DOAC. There was a significant difference between low-dose dabigatran and low-dose apixaban with regard to stroke/SE (HR [95\%CI]: 0.53 [0.30-0.93]) and the safety composite $(2.02[1.42-2.86])$ but not the benefit/risk composite (0.96 [0.75-1.22]). The HR was $0.43([0.26-0.71)$ for all-cause mortality and $0.49([0.34-0.71])$ for the effectiveness composite. The HR for gastrointestinal bleeding was 2.47 ([1.47-4.16]).

There were no significant differences between of low-dose rivaroxaban and low-dose apixaban with regard to stroke/SE (HR: $0.70 ;[0.41-1.17])$ or the benefit/risk composite (1.06 ([0.84-1.35]) but rivaroxaban presented a worse safety profile (1.58 (1.09-2.29]). When comparing low-dose dabigatran with low-dose rivaroxaban, we did not find significant differences for stroke/SE (HR: 0.80; [0.40-1.59]), the safety composite (HR: 1.16; $[0.79-1.72])$, or the benefit/risk composite (HR: 0.96; $[0.72-1.28])$.

\section{Sensitivity Analyses}

\section{The Intent-To-Treat Analysis}

Intention-to-treat (ITT) analyses of the IPTW populations followed up for 365 days gave consistent results (Supplementary Tables S7.1, S7.2) for all comparisons vs. warfarin or other DOACs.

\section{The Impact of Unmeasured Confounders}

For dabigatran vs. warfarin, the E-value corresponding to the CI boundary closest to 1 for the risk of death was 2.21 (Table 3). The observed HR for death might have been due to an unmeasured confounder that occurred 2.21 times more often in the dabigatran group than in the warfarin group and thus increased the death rate by a factor of 2 . This assumes no correlation between the unmeasured confounder and the measured confounders used in the propensity score.

The E-value corresponding to the CI boundary closest to 1 for the various comparisons ranged from 1.36 to 3.62. Lastly, the E-value corresponding to the HR point estimates for the various comparisons ranged from 2.28 to 3.77 -indicating that these situations are less likely to occur. 


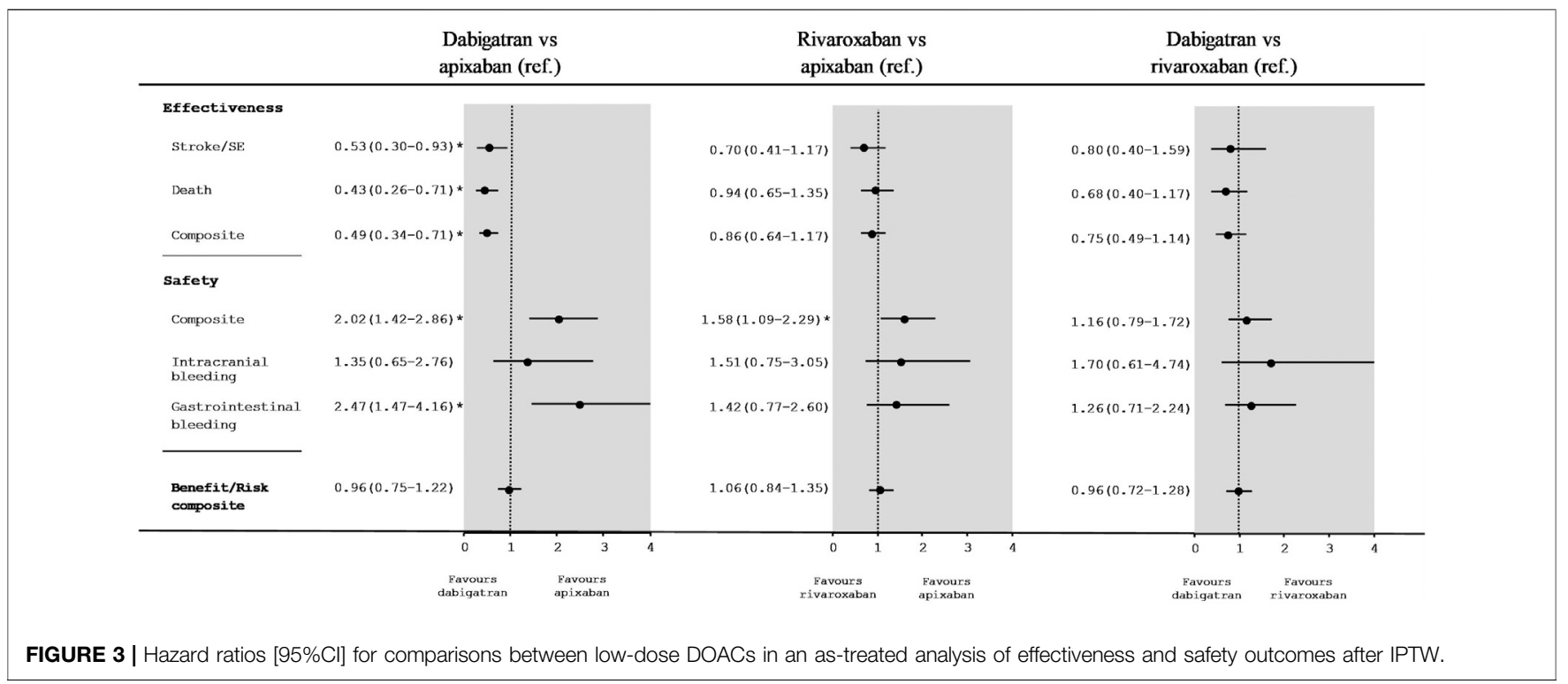

TABLE 3 | E-values for significant comparisons (as-treated analysis) of low-dose DOACs with warfarin and with each other.

Hazard ratio $(95 \% \mathrm{Cl})$

\section{E-value corresponding to the $\mathrm{Cl}$ boundary closest to 1}

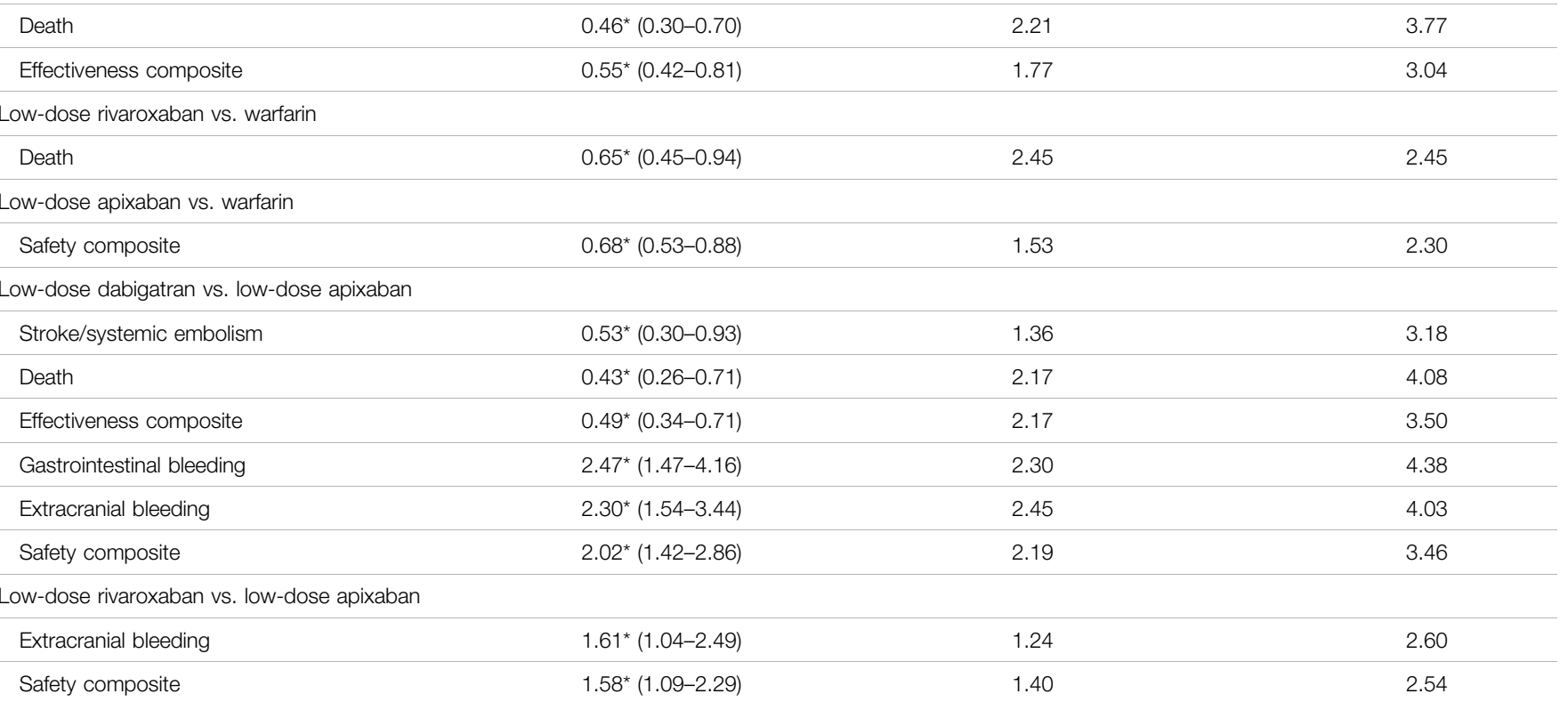

Low-dose dabigatran vs. warfarin

\section{The Negative Control and Impact of the Temporal Trends}

With regard to the rate per 100 person-years of pneumonia vs. warfarin and DOAC (Supplementary Table S8), none of the comparisons gave a significant HR. Moreover, the rates per 100 person-years of hospitalization for diabetes complications were quite similar for warfarin and DOACs, with no significant HRs. As expected, the results were similar in all the groups.
Similar results were observed for the overall comparative effectiveness and safety of each low-dose of DOACs versus warfarin (Supplementary Table S7.3) and also each low-dose of DOACs versus each other (Supplementary Table S7.4) with the inclusion of the base date of cohort entry in the IPTW matching. Some outcomes were marginally modified for the comparison versus warfarin mainly for rivaroxaban safety composite. 


\section{DISCUSSION}

\section{Low-Dose Direct Oral Anticoagulants Compared With Warfarin}

In our population-based study, we did not observe a significant reduction in the risk of the primary outcome (stroke/SE) for any of the low-dose DOACs (dabigatran, rivaroxaban, and apixaban) vs. warfarin. Moreover, there were no significant relationships with the safety profile, except for low-dose apixaban vs. warfarin (a 32\% risk reduction for apixaban). With regard to the secondary outcomes, low-dose dabigatran and low-dose rivaroxaban were associated with a reduction (vs. warfarin) in the risk of all-cause mortality that ranged from 35 to $54 \%$.

Our effectiveness and safety results for patients using low-dose dabigatran or warfarin are quite similar to those published for the RE-LY study (Connolly et al., 2009). Although a number of observational studies have compared dabigatran, rivaroxaban, and apixaban with warfarin in terms of effectiveness and safety, (Graham et al., 2016; Larsen et al., 2016; Hernandez et al., 2017; Nielsen et al., 2017; Li et al., 2018; Lopes et al., 2018; Graham et al., 2019) but few reported on the impact of low dose levels. However, Li et al. evaluated the effectiveness and safety of different dose levels of apixaban (vs. warfarin) with a similar study design and in a similar patient population. Apixaban $2.5 \mathrm{mg}$ twice daily was associated with a lower risk of major bleeding (HR [95\%CI]: 0.59 [0.49-0.71]) (Li et al., 2018). Our results are also consistent with those of another similar study in which (relative to warfarin) low-dose apixaban and low-dose dabigatran had no significant effects on a stroke/SE outcome, low-dose dabigatran was associated with a reduction in the risk of death, and low-dose apixaban presented a better safety profile for bleeding events (Rahme et al., 2021).

\section{Low-Dose Direct Oral Anticoagulants Compared With Each Other}

Low-dose dabigatran presented a $47 \%$ difference in stroke/SE when compared with apixaban; however, it also had a less favorable safety profile, with more than a two-fold relative increase in the major bleeding risk. For low-dose rivaroxaban vs apixaban, we did not observe a significant difference in stroke/SE, although low-dose rivaroxaban had a less favorable safety composite. We noted no significant difference in the comparison of dabigatran and rivaroxaban for the effectiveness and safety outcomes.

The published RCTs did not perform head-to-head comparisons of different dose levels of DOACs. Furthermore, the observational studies of effectiveness and safety compared full dose levels of dabigatran, rivaroxaban, and apixaban-the three most widely used DOACs (Graham et al., 2019; Bonde et al., 2020; Fralick et al., 2020) A recent meta-analysis reported indirect comparisons, although the data on low-dose DOACs were scarce ( $\mathrm{Li}$ et al., 2019). There were no significant differences in the stroke/SE outcome for rivaroxaban or dabigatran, when compared with apixaban. However, the risk of major bleeding was significantly higher for rivaroxaban than for apixaban (HR [95\%CI]: 1.71 [1.51-1.94]). Moreover, a recent study reported nonsignificant differences in the stroke/ SE outcome between low doses of dabigatran, rivaroxaban, and apixaban; however, apixaban had a better safety profile (Durand et al., 2021).

A recent placebo-controlled RCT in older Japanese patients with non-valvular AF (where a standard dose is not appropriate) found that edoxaban was efficacious in preventing stroke/SE and did not have any impact on major bleeding (other than gastrointestinal bleeding) (Okumura et al., 2020). In view of the lack of RCT data and the high prevalence of low-dose DOAC use, further studies of the effectiveness and safety of low-dose DOACs are clearly warranted. Moreover, given that the net benefit seems to vary from one DOAC to another, pharmacokinetic data for specific populations (such as those with higher risks of thrombosis and bleeding) must be generated by comparing plasma drug levels and factor Xa inhibition as a function of the dose level and the outcomes (Testa et al., 2018; Sukumar et al., 2019).

Our study had a number of strengths, including the large sample size and the analyses of the relative effectiveness and safety of lowdosage DOACs vs. warfarin and other DOACs in patients with AF. We assessed several clinical outcomes, in order to balance the overall benefits and risks. We used an IPTW population score model to build cohorts that were well balanced at baseline with regard to relevant factors, and we also performed several sensitivity analyses.

Our study also had some limitations. Firstly, this observational study was based on administrative data and so might have been subject to confounding bias by unadjusted factors (blood pressure control, laboratory values, international normalized ratio control, body weight, and estimated glomerular filtration rate) or to residual channeling bias. Secondly, most of our patients were older and ethnically white, and so our present results might not be generalizable to other patient settings (e.g., non-hospitalized individuals with $\mathrm{AF}$ ), other age groups, or other ethnic groups (Shen et al., 2007). Lastly, residual bias is still possible-especially with regard to unmeasured variables and the healthy population effect.

The results of this population-based study suggest that low-dose dabigatran has a better effective composite than warfarin. Compared with apixaban, low-dose dabigatran had a better effectiveness composite but a worse safety profile. Low-dose apixaban had a better safety composite than warfarin and other low-dose DOACs. Studies of plasma drug levels and factor Xa inhibition as a function of the dose level and outcomes are now warranted, since the net benefit appears to vary from one DOAC to another.

\section{DATA AVAILABILITY STATEMENT}

The original contributions presented in the study are included in the article/Supplementary Material, further inquiries can be directed to the corresponding author.

\section{ETHICS STATEMENT}

The studies involving human participants were reviewed and approved by Ethics committee at the University of Montreal. Written informed consent for participation was not required for this study in accordance with the national legislation and the institutional requirements. 


\section{AUTHOR CONTRIBUTIONS}

All authors listed have made a substantial, direct, and intellectual contribution to the work and approved it for publication.

\section{FUNDING}

The study was funded by the Heart and Stroke Foundation of Canada (G-17-0018326) and the Réseau Québécois de Recherche sur le Médicament (RQRM).

\section{REFERENCES}

Allan, V., Ramagopalan, S. V., Mardekian, J., Jenkins, A., Li, X., Pan, X., et al. (2020). Propensity Score Matching and Inverse Probability of Treatment Weighting to Address Confounding by Indication in Comparative Effectiveness Research of Oral Anticoagulants. J. Comp. Eff. Res. 9 (9), 603-614. doi:10.2217/cer-2020-0013

Austin, P. C., and Stuart, E. A. (2015). Moving towards Best Practice when Using Inverse Probability of Treatment Weighting (IPTW) Using the Propensity Score to Estimate Causal Treatment Effects in Observational Studies. Stat. Med. 34 (28), 3661-3679. doi:10.1002/sim.6607

Blais, C. L. L., Hamel, D., Brown, K., Rinfret, S., Cartier, R., Giguère, M., et al. (2012). Évaluation des soins et surveillance des maladies cardiovasculaires de santé publique du Québec et de L'institut national d'excellence en santé et services sociaux, Gouvernement du Québec, Institut national de santé publique, Institut national d'excellence en santé et des services sociaux 1-9.

Bonde, A. N., Martinussen, T., Lee, C. J., Lip, G. Y. H., Staerk, L., Bang, C. N., et al. (2020). Rivaroxaban versus Apixaban for Stroke Prevention in Atrial Fibrillation: An Instrumental Variable Analysis of a Nationwide Cohort. Circ. Cardiovasc. Qual. Outcomes 13 (4), e006058. doi:10.1161/ CIRCOUTCOMES.119.006058

Colilla, S., Crow, A., Petkun, W., Singer, D. E., Simon, T., and Liu, X. (2013). Estimates of Current and Future Incidence and Prevalence of Atrial Fibrillation in the U.S. Adult Population. Am. J. Cardiol. 112 (8), 1142-1147. doi:10.1016/ j.amjcard.2013.05.063

Connolly, S. J., Ezekowitz, M. D., Yusuf, S., Eikelboom, J., Oldgren, J., Parekh, A., et al. (2009). Dabigatran versus Warfarin in Patients with Atrial Fibrillation. N. Engl. J. Med. 361 (12), 1139-1151. doi:10.1056/NEJMoa0905561

Connolly, S. J., Ezekowitz, M. D., Yusuf, S., Reilly, P. A., and Wallentin, L. (2010). Newly Identified Events in the RE-LY Trial. N. Engl. J. Med. 363 (19), 1875-1876. doi:10.1056/NEJMc1007378

Crane, S. J., Tung, E. E., Hanson, G. J., Cha, S., Chaudhry, R., and Takahashi, P. Y. (2010). Use of an Electronic Administrative Database to Identify Older Community Dwelling Adults at High-Risk for Hospitalization or Emergency Department Visits: the Elders Risk Assessment index. BMC Health Serv. Res. 10, 338. doi:10.1186/1472-6963-10-338

Culebras, A., and Messé, S. R. (2014). Summary of Evidence-Based Guideline Update: Prevention of Stroke in Nonvalvular Atrial Fibrillation: Report of the Guideline Development Subcommittee of the American Academy of Neurology. Neurology 83 (13), 1220. doi:10.1212/WNL.0000000000000831

Cutler, T. W., Chuang, A., Huynh, T. D., Witt, R. G., Branch, J., Pon, T., et al. (2014). A Retrospective Descriptive Analysis of Patient Adherence to Dabigatran at a Large Academic Medical center. J. Manag. Care Spec. Pharm. 20 (10), 1028-1034. doi:10.18553/jmcp.2014.20.10.1028

D'Hoore, W., Bouckaert, A., and Tilquin, C. (1996). Practical Considerations on the Use of the Charlson Comorbidity index with Administrative Data Bases. J. Clin. Epidemiol. 49 (12), 1429-1433. doi:10.1016/s0895-4356(96)00271-5

Deyo, R. A., Cherkin, D. C., and Ciol, M. A. (1992). Adapting a Clinical Comorbidity index for Use with ICD-9-CM Administrative Databases. J. Clin. Epidemiol. 45 (6), 613-619. doi:10.1016/0895-4356(92)90133-8

\section{ACKNOWLEDGMENTS}

We thank the RAMQ and Quebec Health Ministry for assistance with data handling and the Commission d'accès à l'information for authorizing the study.

\section{SUPPLEMENTARY MATERIAL}

The Supplementary Material for this article can be found online at: https://www.frontiersin.org/articles/10.3389/fphar.2021.812018/ full\#supplementary-material

Durand, M., Schnitzer, M. E., Pang, M., Carney, G., Eltonsy, S., Filion, K. B., et al. (2021). Effectiveness and Safety Among Direct Oral Anticoagulants in Nonvalvular Atrial Fibrillation: A Multi-Database Cohort Study with MetaAnalysis. Br. J. Clin. Pharmacol. 87 (6), 2589-2601. doi:10.1111/bcp.14669

Eguale, T., Winslade, N., Hanley, J. A., Buckeridge, D. L., and Tamblyn, R. (2010). Enhancing Pharmacosurveillance with Systematic Collection of Treatment Indication in Electronic Prescribing: a Validation Study in Canada. Drug Saf. 33 (7), 559-567. doi:10.2165/11534580-000000000-00000

Fanikos, J., Grasso-Correnti, N., Shah, R., Kucher, N., and Goldhaber, S. Z. (2005). Major Bleeding Complications in a Specialized Anticoagulation Service. Am. J. Cardiol. 96 (4), 595-598. doi:10.1016/j.amjcard.2005.03.104

Fillion, V., Sirois, M. J., Gamache, P., Guertin, J. R., Morin, S. N., and Jean, S. (2019). Frailty and Health Services Use Among Quebec Seniors with Non-hip Fractures: a Population-Based Study Using Adminsitrative Databases. BMC Health Serv. Res. 19 (1), 70. doi:10.1186/s12913-019-3865-Z

Fralick, M., Colacci, M., Schneeweiss, S., Huybrechts, K. F., Lin, K. J., and Gagne, J. J. (2020). Effectiveness and Safety of Apixaban Compared with Rivaroxaban for Patients with Atrial Fibrillation in Routine Practice: A Cohort Study. Ann. Intern. Med. 172 (7), 463-473. doi:10.7326/M19-2522

Friberg, L., Rosenqvist, M., and Lip, G. Y. (2012). Evaluation of Risk Stratification Schemes for Ischaemic Stroke and Bleeding in 182678 Patients with Atrial Fibrillation: the Swedish Atrial Fibrillation Cohort Study. Eur. Heart J. 33 (12), 1500-1510. doi:10.1093/eurheartj/ehr488

Graham, D. J., Baro, E., Zhang, R., Liao, J., Wernecke, M., Reichman, M. E., et al. (2019). Comparative Stroke, Bleeding, and Mortality Risks in Older Medicare Patients Treated with Oral Anticoagulants for Nonvalvular Atrial Fibrillation. Am. J. Med. 132 (5), 596-e11. doi:10.1016/j.amjmed.2018.12.023

Graham, D. J., Reichman, M. E., Wernecke, M., Hsueh, Y. H., Izem, R., Southworth, M. R., et al. (2016). Stroke, Bleeding, and Mortality Risks in Elderly Medicare Beneficiaries Treated with Dabigatran or Rivaroxaban for Nonvalvular Atrial Fibrillation. JAMA Intern. Med. 176 (11), 1662-1671. doi:10.1001/ jamainternmed.2016.5954

Granger, C. B., Alexander, J. H., McMurray, J. J., Lopes, R. D., Hylek, E. M., Hanna, M., et al. (2011). Apixaban versus Warfarin in Patients with Atrial Fibrillation. N. Engl. J. Med. 365 (11), 981-992. doi:10.1056/ NEJMoa1107039

Hart, R. G., Pearce, L. A., and Aguilar, M. I. (2007). Meta-analysis: Antithrombotic Therapy to Prevent Stroke in Patients Who Have Nonvalvular Atrial Fibrillation. Ann. Intern. Med. 146 (12), 857-867. doi:10.7326/0003-4819146-12-200706190-00007

Hernandez, I., Zhang, Y., and Saba, S. (2017). Comparison of the Effectiveness and Safety of Apixaban, Dabigatran, Rivaroxaban, and Warfarin in Newly Diagnosed Atrial Fibrillation. Am. J. Cardiol. 120 (10), 1813-1819. doi:10.1016/j.amjcard.2017.07.092

Humphries, K. H., Jackevicius, C., Gong, Y., Svensen, L., Cox, J., Tu, J. V., et al. (2004). Population Rates of Hospitalization for Atrial Fibrillation/flutter in Canada. Can. J. Cardiol. 20 (9), 869-876.

January, C. T., Wann, L. S., Alpert, J. S., Calkins, H., Cigarroa, J. E., Cleveland, J. C., et al. (2014). 2014 AHA/ACC/HRS Guideline for the Management of Patients with Atrial Fibrillation: a Report of the American College of Cardiology/ American Heart Association Task Force on Practice Guidelines and the 
Heart Rhythm Society. J. Am. Coll. Cardiol. 64 (21), e1-76. doi:10.1016/ j.jacc.2014.03.022

Jensen, P. N., Johnson, K., Floyd, J., Heckbert, S. R., Carnahan, R., and Dublin, S. (2012). A Systematic Review of Validated Methods for Identifying Atrial Fibrillation Using Administrative Data. Pharmacoepidemiol. Drug Saf. 21 (Suppl. 1), 141-147. doi:10.1002/pds.2317

Larsen, T. B., Skjøth, F., Nielsen, P. B., Kjældgaard, J. N., and Lip, G. Y. (2016). Comparative Effectiveness and Safety of Non-vitamin K Antagonist Oral Anticoagulants and Warfarin in Patients with Atrial Fibrillation: Propensity Weighted Nationwide Cohort Study. BMJ 353, i3189. doi:10.1136/bmj.i3189

Levy, A. R., Tamblyn, R. M., Fitchett, D., McLeod, P. J., and Hanley, J. A. (1999). Coding Accuracy of Hospital Discharge Data for Elderly Survivors of Myocardial Infarction. Can. J. Cardiol. 15 (11), 1277-1282.

Li, G., Lip, G. Y. H., Holbrook, A., Chang, Y., Larsen, T. B., Sun, X., et al. (2019). Direct Comparative Effectiveness and Safety between Non-vitamin K Antagonist Oral Anticoagulants for Stroke Prevention in Nonvalvular Atrial Fibrillation: a Systematic Review and Meta-Analysis of Observational Studies. Eur. J. Epidemiol. 34 (2), 173-190. doi:10.1007/ s10654-018-0415-7

Li, X., Keshishian, A., Hamilton, M., Horblyuk, R., Gupta, K., Luo, X., et al. (2018). Apixaban 5 and $2.5 \mathrm{Mg}$ Twice-Daily versus Warfarin for Stroke Prevention in Nonvalvular Atrial Fibrillation Patients: Comparative Effectiveness and Safety Evaluated Using a Propensity-Score-Matched Approach. PLoS One 13 (1), e0191722. doi:10.1371/journal.pone.0191722

Lip, G. Y., Nieuwlaat, R., Pisters, R., Lane, D. A., and Crijns, H. J. (2010). Refining Clinical Risk Stratification for Predicting Stroke and Thromboembolism in Atrial Fibrillation Using a Novel Risk FactorBased Approach: the Euro Heart Survey on Atrial Fibrillation. Chest 137 (2), 263-272. doi:10.1378/chest.09-1584

Lip, G. Y. H., Banerjee, A., Boriani, G., Chiang, C. E., Fargo, R., Freedman, B., et al. (2018). Antithrombotic Therapy for Atrial Fibrillation: CHEST Guideline and Expert Panel Report. Chest 154 (5), 1121-1201. doi:10.1016/j.chest.2018.07.040

Lopes, R. D., Steffel, J., Di Fusco, M., Keshishian, A., Luo, X., Li, X., et al. (2018). Effectiveness and Safety of Anticoagulants in Adults with Non-valvular Atrial Fibrillation and Concomitant Coronary/Peripheral Artery Disease. Am. J. Med. 131 (9), 1075-e4. doi:10.1016/j.amjmed.2018.05.007

Maura, G., Pariente, A., Alla, F., and Billionnet, C. (2017). Adherence with Direct Oral Anticoagulants in Nonvalvular Atrial Fibrillation New Users and Associated Factors: a French Nationwide Cohort Study. Pharmacoepidemiol. Drug Saf. 26 (11), 1367-1377. doi:10.1002/pds.4268

McGrath, E. R., Kapral, M. K., Fang, J., Eikelboom, J. W., Conghaile, Aó., O'Conghaile, A., et al. (2013). Association of Atrial Fibrillation with Mortality and Disability after Ischemic Stroke. Neurology 81 (9), 825-832. doi:10.1212/WNL.0b013e3182a2cc15

Miyasaka, Y., Barnes, M. E., Gersh, B. J., Cha, S. S., Bailey, K. R., Abhayaratna, W. P., et al. (2006). Secular Trends in Incidence of Atrial Fibrillation in Olmsted County, Minnesota, 1980 to 2000, and Implications on the Projections for Future Prevalence. Circulation 114 (2), 119-125. doi:10.1161/ CIRCULATIONAHA.105.595140

Nielsen, P. B., Skjøth, F., Søgaard, M., Kjældgaard, J. N., Lip, G. Y., and Larsen, T. B. (2017). Effectiveness and Safety of Reduced Dose Non-vitamin K Antagonist Oral Anticoagulants and Warfarin in Patients with Atrial Fibrillation: Propensity Weighted Nationwide Cohort Study. BMJ 356, j510. doi:10.1136/ bmj.j510

Okumura, K., Akao, M., Yoshida, T., Kawata, M., Okazaki, O., Akashi, S., et al. (2020). Low-Dose Edoxaban in Very Elderly Patients with Atrial Fibrillation. $N$. Engl. J. Med. 383 (18), 1735-1745. doi:10.1056/NEJMoa2012883

Pampalon, R., Hamel, D., Gamache, P., and Raymond, G. (2009). A Deprivation index for Health Planning in Canada. Chronic Dis. Can. 29 (4), 178-191. doi:10.24095/hpcdp.29.4.05

Patel, M. R., Mahaffey, K. W., Garg, J., Pan, G., Singer, D. E., Hacke, W., et al. (2011). Rivaroxaban versus Warfarin in Nonvalvular Atrial Fibrillation. N. Engl. J. Med. 365 (10), 883-891. doi:10.1056/NEJMoa1009638

Perreault, S., de Denus, S., White-Guay, B., Côté, R., Schnitzer, M. E., Dubé, M. P., et al. (2020). Oral Anticoagulant Prescription Trends, Profile Use, and
Determinants of Adherence in Patients with Atrial Fibrillation. Pharmacotherapy 40 (1), 40-54. doi:10.1002/phar.2350

Perreault, S., Shahabi, P., Cote, R., Dumas, S., Rouleau-Mailloux, É., Feroz Zada, Y., et al. (2018). Rationale, Design, and Preliminary Results of the Quebec Warfarin Cohort Study. Clin. Cardiol. 41 (5), 576-585. doi:10.1002/clc.22948

Pisters, R., Lane, D. A., Nieuwlaat, R., de Vos, C. B., Crijns, H. J., and Lip, G. Y. (2010). A Novel User-Friendly Score (HAS-BLED) to Assess 1-year Risk of Major Bleeding in Patients with Atrial Fibrillation: the Euro Heart Survey. Chest 138 (5), 1093-1100. doi:10.1378/chest.10-0134

Rahme, E., Godin, R., Nedjar, H., Dasgupta, K., and Tagalakis, V. (2021). Dose Specific Effectiveness and Safety of DOACs in Patients with Non-valvular Atrial Fibrillation: A Canadian Retrospective Cohort Study. Thromb. Res. 203, 121-130. doi:10.1016/j.thromres.2021.05.005

Roy, L., Zappitelli, M., White-Guay, B., Lafrance, J. P., Dorais, M., and Perreault, S. (2020). Agreement between Administrative Database and Medical Chart Review for the Prediction of Chronic Kidney Disease G Category. Can. J. Kidney Health Dis. 7, 2054358120959908. doi:10.1177/ 2054358120959908

Samsa, G. P., and Matchar, D. B. (2000). Relationship between Test Frequency and Outcomes of Anticoagulation: a Literature Review and Commentary with Implications for the Design of Randomized Trials of Patient SelfManagement. J. Thromb. Thrombolysis 9 (3), 283-292. doi:10.1023/a: 1018778914477

Shen, A. Y., Yao, J. F., Brar, S. S., Jorgensen, M. B., and Chen, W. (2007). Racial/ ethnic Differences in the Risk of Intracranial Hemorrhage Among Patients with Atrial Fibrillation. J. Am. Coll. Cardiol. 50 (4), 309-315. doi:10.1016/ j.jacc.2007.01.098

Steinberg, B. A., Holmes, D. N., Piccini, J. P., Ansell, J., Chang, P., Fonarow, G. C., et al. (2013). Early Adoption of Dabigatran and its Dosing in US Patients with Atrial Fibrillation: Results from the Outcomes Registry for Better Informed Treatment of Atrial Fibrillation. J. Am. Heart Assoc. 2 (6), e000535. doi:10.1161/ JAHA.113.000535

Sukumar, S., Gulilat, M., Linton, B., Gryn, S. E., Dresser, G. K., Alfonsi, J. E., et al. (2019). Apixaban Concentrations with Lower Than Recommended Dosing in Older Adults with Atrial Fibrillation. J. Am. Geriatr. Soc. 67 (9), 1902-1906. doi:10.1111/jgs. 15982

Tamblyn, R., Lavoie, G., Petrella, L., and Monette, J. (1995). The Use of Prescription Claims Databases in Pharmacoepidemiological Research: the Accuracy and Comprehensiveness of the Prescription Claims Database in Québec. J. Clin. Epidemiol. 48 (8), 999-1009. doi:10.1016/0895-4356(94) 00234-h

Tamblyn, R., Reid, T., Mayo, N., McLeod, P., and Churchill-Smith, M. (2000). Using Medical Services Claims to Assess Injuries in the Elderly: Sensitivity of Diagnostic and Procedure Codes for Injury Ascertainment. J. Clin. Epidemiol. 53 (2), 183-194. doi:10.1016/s0895-4356(99)00136-5

Testa, S., Paoletti, O., Legnani, C., Dellanoce, C., Antonucci, E., Cosmi, B., et al. (2018). Low Drug Levels and Thrombotic Complications in High-Risk Atrial Fibrillation Patients Treated with Direct Oral Anticoagulants. J. Thromb. Haemost. 16 (5), 842-848. doi:10.1111/jth.14001

Thigpen, J. L., Dillon, C., Forster, K. B., Henault, L., Quinn, E. K., Tripodis, Y., et al. (2015). Validity of International Classification of Disease Codes to Identify Ischemic Stroke and Intracranial Hemorrhage Among Individuals with Associated Diagnosis of Atrial Fibrillation. Circ. Cardiovasc. Qual. Outcomes 8 (1), 8-14. doi:10.1161/ CIRCOUTCOMES.113.000371

Tirschwell, D. L., and Longstreth, W. T., Jr. (2002). Validating Administrative Data in Stroke Research. Stroke 33 (10), 2465-2470. doi:10.1161/ 01.str.0000032240.28636.bd

VanderWeele, T. J., and Ding, P. (2017). Sensitivity Analysis in Observational Research: Introducing the E-Value. Ann. Intern. Med. 167 (4), 268-274. doi:10.7326/M16-2607

Wilchesky, M., Tamblyn, R. M., and Huang, A. (2004). Validation of Diagnostic Codes within Medical Services Claims. J. Clin. Epidemiol. 57 (2), 131-141. doi:10.1016/S0895-4356(03)00246-4

Xu, H., Ruff, C. T., Giugliano, R. P., Murphy, S. A., Nordio, F., Patel, I., et al. (2016). Concomitant Use of Single Antiplatelet Therapy with Edoxaban or Warfarin in 
Patients with Atrial Fibrillation: Analysis from the ENGAGE AF-TIMI48 Trial. J. Am. Heart Assoc. 5 (2), e002587. doi:10.1161/JAHA.115.002587

Conflict of Interest: Author MD was employed by StatSciences Inc.

The remaining authors declare that the research was conducted in the absence of any commercial or financial relationships that could be construed as a potential conflict of interest.

Publisher's Note: All claims expressed in this article are solely those of the authors and do not necessarily represent those of their affiliated organizations, or those of the publisher, the editors, and the reviewers. Any product that may be evaluated in this article, or claim that may be made by its manufacturer, is not guaranteed or endorsed by the publisher.

Copyright (๑ 2022 Perreault, Dragomir, Côté, Lenglet, de Denus, Dorais, WhiteGuay, Brophy, Schnitzer, Dubé and Tardif. This is an open-access article distributed under the terms of the Creative Commons Attribution License (CC BY). The use, distribution or reproduction in other forums is permitted, provided the original author(s) and the copyright owner(s) are credited and that the original publication in this journal is cited, in accordance with accepted academic practice. No use, distribution or reproduction is permitted which does not comply with these terms. 